Papers

\title{
Thermal Behavior of Visible AlGaInP-GaInP Ridge Laser Diodes
}

\author{
Olivier J. F. Martin, Gian-Luca Bona, and Peter Wolf
}

\begin{abstract}
The thermal behavior of visible AIGaInP-GaInP ridge laser diodes is investigated numerically and experimentally. It is shown that various parameters critically influence the thermal resistance $R$ of such devices. $R$ is inversely proportional to the thermal conductivity of the heatsink. A substantial improvement in $R$-quite larger than in the AIGaAs system-is achieved for junction-side-down mounting compared to junction-side-up. $R$ depends strongly on the width $w$ of the ridge and this effect is different for junction-side-up or down mounting. In the first case, $R \sim \log (w)$ and in the second, $R \sim 1 / w$. The thickness of the soldering material is a sensitive parameter which may increase the value of $R$ by up to $15 \mathrm{~K} / \mathrm{W}$. On the other hand, for junction-side-up mounted devices, the top metallization layer has a very favorable effect: a $1 \mu \mathrm{m}$ thick gold layer reduces $R$ by $30 \%$. The dynamics of thermal phenomena is also studied. It is shown that when a laser is switched on the steady state is reached in the ms time range. Finally, our experimental results show a very good agreement with our numerical data.
\end{abstract}

\section{INTRODUCTION}

$\mathrm{T}$ $\mathrm{HE}$ temperature rise during $\mathrm{CW}$ operation of visible laser diodes is a very critical parameter since it strongly affects the device characteristics and reliability. For AlGaAs-GaAs lasers, special attention has already been paid to the thermal phenomena in order to improve device performance and lifetime [1]-[8]. In the AlGaInPGaInP material system, heating of the laser is an even more severe handicap [9], [10].

First, the thermal conductivity of the quaternary cladding layers is considerably lower than for AlGaAs. Second, the threshold current is relatively high compared to AlGaAs-GaAs devices. Therefore, joule heating due to the series resistance causes an additional temperature rise. Third, the temperature sensitivity parameter $T_{0}$ of the laser threshold is still rather low and is typically $100 \mathrm{~K}$ for devices lasing at $670 \mathrm{~nm}$. Fourth, it has been reported that degradation mechanisms in visible lasers are also strongly affected by the operating temperature, e.g., operation above $60^{\circ} \mathrm{C}$ reduces the lifetime substantially [11].

Therefore, it is clear that for high-power operation of

Manuscript received February 5, 1991; revised January 25, 1992.

The authors are with Zurich Research Laboratory, IBM Research Division, $\mathrm{CH}-8803$ Rüschlikon, Switzerland.

IEEE Log Number 9203204 visible laser diodes in the transverse fundamental mode, such as required for optical storage, an efficient heatsinking and a good understanding of the thermal phenomena of the device are mandatory. However, to the best of our knowledge, only limited data on the thermal behavior of AlGaInP-GaInP devices are available today. Hatakoshi et al. [12] have studied the influence of the cladding layer on the thermal behavior of such lasers. In the present paper, we investigate other parameters such as the heatsinking and soldering materials, the active region width, the top metallization and we also give results on the dynamic behavior of thermal effects in AlGaInP-GaInP lasers.

\section{Numerical Model}

In the model, we assume that heat is uniformly generated within the active region and we neglect any variation of heat production along the laser cavity (heating of the mirrors, longitudinal variation of carrier density, etc.). Therefore, we assume that heat flow is homogeneous along the structure and two-dimensional at any cross section of the laser. We also neglect any radiation and convection losses. This approximation is valid as long as the surface temperature of the device remains low.

From a theoretical point of view, our problem involves the solution of the heat equation

$$
\rho \cdot C_{p} \frac{\partial T}{\partial t}=\vec{\nabla}(k \cdot \vec{\nabla} T)+Q
$$

over a two-dimensional cross section of the laser, where $T$ is the temperature, $\rho$ is the density, $C_{p}$ is the specific heat, $k$ is the thermal conductivity, and $Q$ is the heat source density. All these physical parameters can vary considerably over the cross section as can be seen in Fig. 1. This figure shows the cross section of an AlGaInPGaInP ridge laser mounted junction-side-down on a heatsink [10]. We have also investigated junction-side-up mounting. In our simulations, we take advantage of the symmetry axis of the structure to reduce calculation time. The values of the thermal conductivity used in the simulations are from Adachi for GaAs [13] and Hatakoshi for AlGaInP [12]. For GaInP we have used the following empirical relation between the thermal resistance $r$ and 


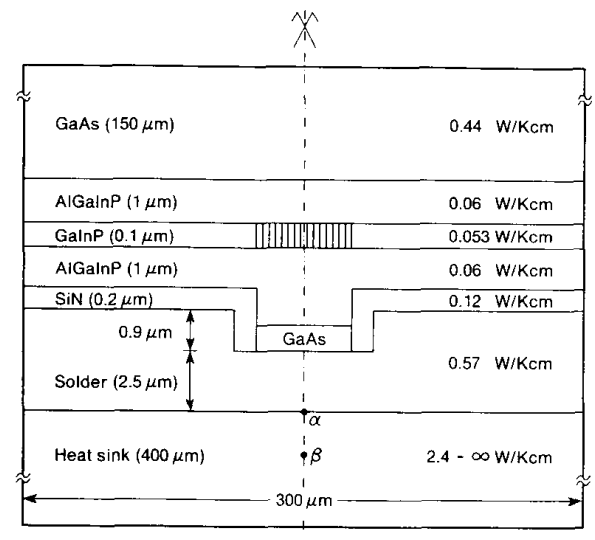

Fig. 1. Schematic structure of AlGaInP-GaInP ridge laser in the "junction-side-down" configuration. The thickness and thermal conductivity of each layer is represented. For the simulations, heat is uniformly generated within the hatched area (active region) which is varied between 5 and $30 \mu \mathrm{m}$.

the Ga content $x: 1 / k=r=r_{0}+\left(r_{1}-r_{0}\right) \cdot x+B$. $(1-x) \cdot x$, with $r_{0}=1.35 \mathrm{cmK} / \mathrm{W}, r_{1}=1.5 \mathrm{cmK} / \mathrm{W}$ and $B=71.5 \mathrm{cmK} / \mathrm{W}$. The conductivity values for $\mathrm{Si}_{3} \mathrm{~N}_{4}$, heatsinking and soldering materials are found in [14][16].

To compute a numerical solution of (1), we discretize this equation over a grid with finite differences and solve the ensuing system of algebraic equations using the alternating direction algorithm [17]. A variable grid is used with a smaller mesh size in the regions of particular interest; the total number of meshes is typically 6000 . Although we are interested in the steady state of the system $(\partial T / \partial t=0)$, we maintain the time dependence in (1) and approximate it also with finite differences. This enables us to simulate the dynamic behavior of the system. At $t=0$ the medium is initialized with uniform temperature (e.g., room temperature); then, as the calculation proceeds, we can follow the heating of the laser due to the sources $Q$. Finally, from the steady-state temperature we can calculate the thermal resistance $\boldsymbol{R}$ of the device.

\section{Results \\ A. Influence of the Heatsinking Material}

We have considered four different heatsinking materials: CMSH $(\mathrm{Cu} 15 \%-W 85 \%, k=2.4 \mathrm{~W} / \mathrm{Kcm})$, copper $(k=3.85 \mathrm{~W} / \mathrm{Kcm})$, diamond $(k=20 \mathrm{~W} / \mathrm{Kcm})$, and an ideal material with infinite conductivity. The thermal resistance of a $300 \mu \mathrm{m}$ long laser with a $10 \mu \mathrm{m}$ wide ridge, mounted on these various materials is depicted in Fig. 2. Both junction-side-up and junction-side-down mounting are investigated.

The thermal resistance is proportional to the inverse of $k$, although there is no large increase between the ideal heatsink and $\mathrm{CMSH}$, the poorest heatsinking material considered here. The difference for junction-side-down mounting between these extreme cases is only a factor of

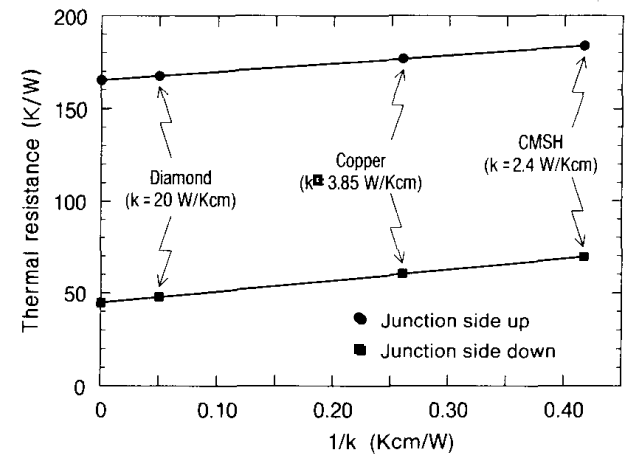

Fig. 2. Dependence of the thermal resistance on the conductivity of the heatsink. The results are for a $300 \mu \mathrm{m}$ long laser with a $10 \mu \mathrm{m}$ wide ridge, bonded with Au $80 \%$-Sn $20 \%$ solder.

1.5 , whereas for junction-side-up it is 1.1 . It is interesting to note that diamond is an almost ideal heatsinking material for lasers since it increases $\boldsymbol{R}$ only by $7 \%$ of the ideal value in the junction-side-down configuration. For junction-side-up, the difference is only $2 \%$, indicating that the GaAs substrate becomes the dominant part of $\boldsymbol{R}$ in this configuration. The two lines in Fig. 2 represent least square fits of the data; they are not parallel because the heat flow in the heatsink is different for junction-up and down. Their slopes are $44 \mathrm{~cm}^{-1}$ for junction-side-up and $59 \mathrm{~cm}^{-1}$ for junction-side-down. These data are in good agreement with our experimental results (see Sections III-B and E) and with values obtained by others [18], [19]. Experimentally, we observed only small differences in $\boldsymbol{R}$ for the different heatsinks. The best results were obtained using diamond and Au $80 \%-$ Sn $20 \%$ solder. However, a copper heatsink with the same solder typically resulted in an increase of $\boldsymbol{R}$ of less than $15 \mathrm{~K} / \mathrm{W}$.

\section{B. Width of the Active Region}

The width of the ridge is an important parameter because it determines the transverse mode of the optical beam. The thermal behavior of the laser also depends critically on this parameter: for a wide ridge, the heat source extends over a broader area. The results of simulations for active region widths between $3.5 \mu \mathrm{m}$ and $30 \mu \mathrm{m}$ are compared in Fig. 3 with experimental results. The behavior of $\boldsymbol{R}$ is different when the laser is mounted junctionside-up than when it is junction-side-down. But in both configurations, narrow ridges are very adverse from a thermal point of view.

For junction-side-up, $\boldsymbol{R}$ is proportional to the logarithm of the width [Fig. 3(a)]. This effect is independent of the heatsinking material which merely shifts the line toward higher resistances for less conductive materials. When the laser is mounted junction-side-down, $\boldsymbol{R}$ is proportional to the inverse of the width of the active region [Fig. 3(b)]. The influence of the heatsinking material is different as well: a poorly conductive material not only shifts the line but also increases its slope slightly. This difference in behavior can be understood by comparing the heat flows in 


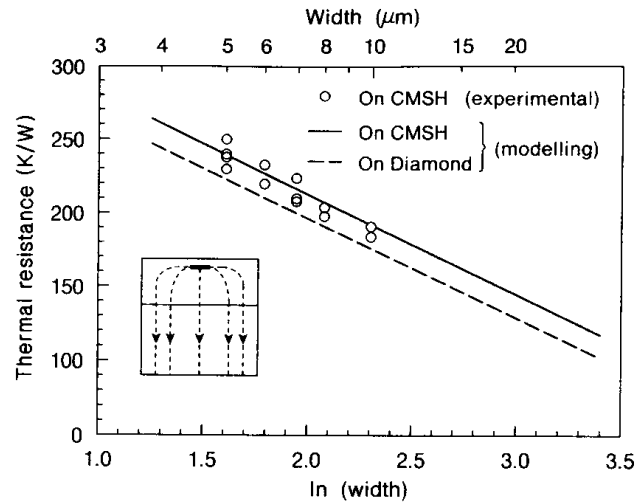

(a)

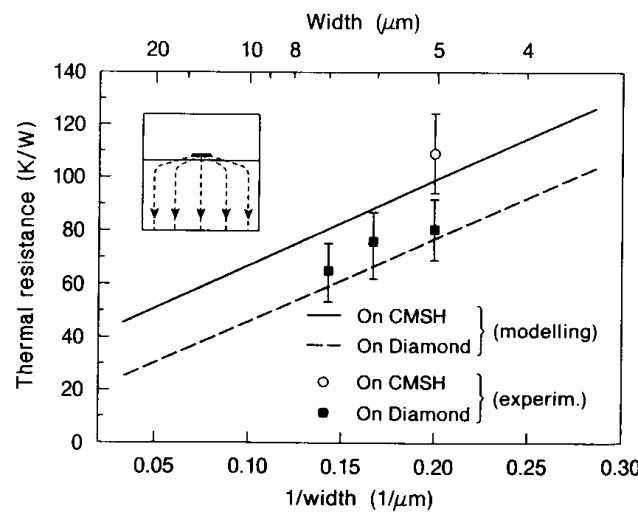

(b)

Fig. 3. Dependence of the thermal resistance on the active region width for two heatsinking materials: (a) junction-side-up and (b) junction-sidedown. The difference in behavior can be explained by the difference of heat flow in the two configurations. A scheme of the corresponding heat flow within the laser is depicted in the insets. The data are calculated for a 300 $\mu \mathrm{m}$ long laser.

the laser obtained from the simulations [see insets in Fig. 3(a) and (b)].

1) For junction-side-up the heat generated in the active region spreads laterally over the entire width of the laser as it flows to the heatsink, leading to a two-dimensional flow in the upper part of the structure, whereas in the heatsink, the flow is vertical and extends over the whole width of the cross section. The association of these two types of heat flows produces the behavior observed in Fig. 3(a):

i) The two-dimensional flow in the upper part of the structure accounts for the logarithmical dependence of $\boldsymbol{R}$ on the width of the active region. Indeed, one can show that in this case, for a homogeneous material of thermal conductivity $k$, the thermal resistance becomes [20]

$$
R=\frac{1}{\pi \cdot k \cdot L} \ln \left(\frac{16 \cdot t}{\pi \cdot w}\right)
$$

This result is valid when the width $w$ of the active region is smaller than the distance $t$ between the active region and the top of the heatsink, which is the case here. $L$ is the length of the laser.

ii) The one-dimensional flow in the heatsink explains that different heatsinking materials simply shift the curves in Fig. 3(a) without affecting their slope. This indicates that the heatsinking material influences the intensity of the heat flow without changing its shape. Therefore the heat flow in the heatsink remains uniform and one-dimensional. This assumption can be verified by writing the thermal resistance as $\boldsymbol{R}=\boldsymbol{R}_{\mathrm{hs}}+\boldsymbol{R}_{\text {laser }}, \boldsymbol{R}_{\text {laser }}$ being the thermal resistance due to the laser and $\boldsymbol{R}_{\mathrm{hs}}$ the contribution of the heatsink. $\boldsymbol{R}_{\text {laser }}$ depends on the width of the active region but is independent of the heatsink material. Thus the difference of thermal resistance for the two heatsinking materials becomes

$$
\Delta \boldsymbol{R}=\boldsymbol{R}_{\mathrm{hs}}^{\mathrm{CMSH}}-\boldsymbol{R}_{\mathrm{hs}}^{\text {diamond }} .
$$

If the heat flow is homogeneous and unidirectional in the heatsink, one can write:

$$
\boldsymbol{R}_{\mathrm{hs}}=\frac{t}{l \cdot w \cdot k}
$$

where $t, l, w$, and $k$ are the thickness, length, width, and thermal conductivity of the heatsink, respectively.

For our configuration, (3) and (4) yield $\Delta R=16.3$ $\mathrm{K} / \mathrm{W}$. The corresponding value obtained from the shift of the curves in Fig. 3(a) is $\Delta \boldsymbol{R}=17.8 \mathrm{~K} / \mathrm{W}$. The good agreement of these two values proves $a$ posteriori our hypothesis of homogeneous unidimensional heat flow in the heatsink.

2) When the laser is mounted junction-side-down, the proximity of the active region to the top of the heatsink strongly influences the heat flow. Owing to the good conductivity of the heatsink material, the heat produced in the active region is rapidly distributed over the whole heatsink width. This leads to a one-dimensional vertical flow in most of the heatsink, which accounts for the behavior of $\boldsymbol{R}$ over the width of the active region.

The expansion of the heat flow in the upper part of the heatsink depends on the heatsinking material. This explains why the slope of the lines in Fig. 3(b) depends on the heatsink material. Although the heat flow is not homogeneous nor unidirectional over the whole heatsink (as it was in the junction-side-up case), the disturbance from such a flow is not very great: the shift between the lines in Fig. 3(b) $(19.6 \mathrm{~K} / \mathrm{W})$ remains close to the value calculated with (4) for a homogeneous heat flow $(16.3 \mathrm{~K} / \mathrm{W})$ and the variation of the slope is less than $5 \%$.

We have used the Paoli method [21] to measure the thermal resistance of our lasers. The values obtained for $300 \mu \mathrm{m}$ long devices with various ridge widths mounted junction-side-up on CMSH heatsinks are shown in Fig. 3(a). The measurements are accurate within $15 \%$ but the error bars have been omitted for clarity. The agreement between experimental and numerical results is excellent. The logarithmical dependence of $\boldsymbol{R}$ on the width of the ridge is clearly observed in the experiments. 
For junction-side-down mounting, we present experimental results for $300 \mu \mathrm{m}$ long devices with ridge widths relevant for high-power, single-mode operation as required for optical storage applications [Fig. 3(b)]. Two different heatsinking materials are investigated experimentally for this configuration. The agreement between experimental and numerical data is excellent and the dependence of $\boldsymbol{R}$ on the inverse of the width of the ridge is also obtained experimentally. We used Au $80 \%-$ Sn $20 \%$ solder for mounting all the lasers presented in this section on their heatsink.

\section{Soldering Material}

We have investigated three different soldering materials: $\mathrm{Au} 80 \%-\mathrm{Sn} 20 \%(k=0.57 \mathrm{~W} / \mathrm{Kcm}), \mathrm{Pb} 40 \%-\mathrm{Sn}$ $60 \%(k=0.42 \mathrm{~W} / \mathrm{Kcm})$, and In $(k=0.62 \mathrm{~W} / \mathrm{Kcm})$. Only the junction-side-down configuration was considered, because the effect of the solder is dominant for this configuration. $\boldsymbol{R}$ as a function of the solder thickness is shown in Fig. 4. For clarity, the results for In are not shown, they are indistinguishable from the $\mathrm{Au}-\mathrm{Sn}$ values.

The solder layer increases the thermal resistance of the laser. But this rise is not simply proportional to the thickness of the solder: it saturates for thick layers. This is due to the influence of the solder layer on the heat flow near the active region. For a thick solder layer $(>3 \mu \mathrm{m})$ the spreading of heat within the layer tends to lower the thermal resistance. Of course this effect is greater with a poorly conducting heatsink (CMSH) than with a good one (diamond). This is also visible in Fig. 4: the saturation of $\boldsymbol{R}$ for a thick solder is more important for CMSH than for the diamond heatsink. Similar results have been reported for AlGaAs lasers [1]. The spreading of heat near the active region may be favored by an additional metal layer between the ridge and the soldering layer. However, this effect is small: we have computed that an additional $3 \mu \mathrm{m}$ thick gold layer decreases $\boldsymbol{R}$ by only $3 \%$.

\section{Heat Spreader}

It is well known that the top metallization on a laser mounted junction-side-up influences its thermal behavior. Therefore, we have studied the effect of a gold layer on top of the laser. Fig. 5 gives the thermal resistance as a function of gold thickness when the laser is mounted on two different heatsinks.

The top metallization strongly reduces the thermal resistance. Most of the improvement is achieved with the first $\mu \mathrm{m}$ of gold: $0.2 \mu \mathrm{m}$ of metal decreases $\boldsymbol{R}$ by $17 \%$ and $0.9 \mu \mathrm{m}$ by $29 \%$. Furthermore a $5 \mu \mathrm{m}$ thick gold spreader reduces $\boldsymbol{R}$ to a value only about $60 \%$ higher than for the junction-side-down configuration.

The gold layer acts like a saturable heat absorber which extracts the heat from the active region and distributes it over the entire width of the laser. It does not affect the heat flow within the heatsink. Therefore the improvement is independent of the heatsinking material and the two curves in Fig. 5 are merely shifted by $17 \mathrm{~K} / \mathrm{W}$.

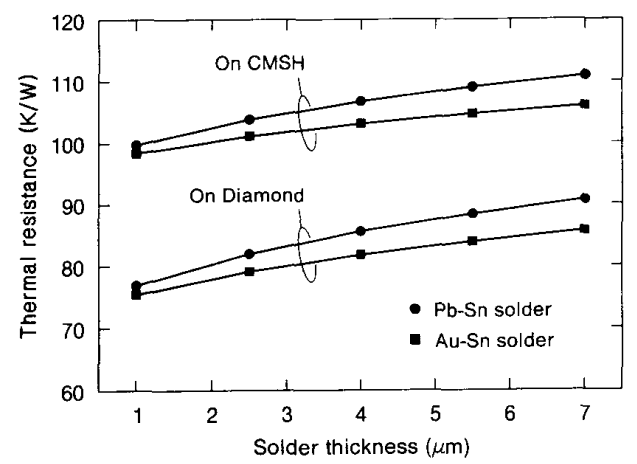

Fig. 4. Thermal resistance versus solder thickness. Two soldering materials: Au $80 \%$-Sn $20 \%(k=0.57 \mathrm{~W} / \mathrm{Kcm})$ and $\mathrm{Pb} 40 \%-\mathrm{Sn} 60 \%(k=$ $0.42 \mathrm{~W} / \mathrm{Kcm})$ are used to bond the laser junction-side-down on two different heatsinks. Results are for a $300 \mu \mathrm{m}$ long laser with a $5 \mu \mathrm{m}$ wide ridge.

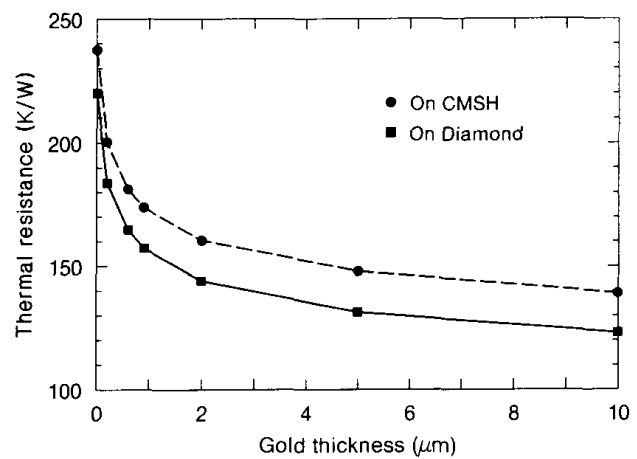

Fig. 5. Thermal resistance versus top gold metallization thickness. The important decrease of $\boldsymbol{R}$ is independent of the heatsinking material. The data are for a $300 \mu \mathrm{m}$ long laser with a $5 \mu \mathrm{m}$ wide ridge, mounted junctionside-up.

It is interesting to point out that the improvement obtained with a top metallization is particularly important for this kind of lasers. This is due to the very poor conductivity of the AlGaInP cladding layers $(k=0.06$ $\mathrm{W} / \mathrm{Kcm}$ ). For example a $0.9 \mu \mathrm{m}$ gold metallization reduces $\boldsymbol{R}$ by only $12 \%$ for an AlGaAs laser [1] and by $19 \%$ for an InP laser [22].

\section{E. Length of the Laser}

The underlying assumption of our two-dimensional model is that the laser cavity extends infinitely. Consequently our model does not take into account thermal effects which might occur at the mirror. In order to evaluate the influence of this drawback on the accuracy of our results, we compare in this section numerical and experimental results for various laser lengths. As a matter of fact, the relative importance of the mirror is larger for short devices than for long ones. Therefore the agreement between numerical and experimental data should be better for long devices.

The values obtained with the Paoli method [21] for various device lengths are presented in Fig. 6. The lasers are 


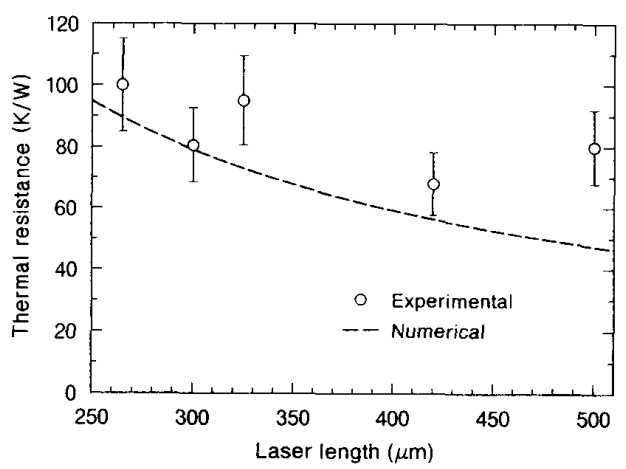

Fig. 6. Thermal resistance as a function of the laser length. The devices are mounted junction-side-down on diamond heatsinks with $\mathrm{Au}-\mathrm{Sn}$ solde and have $5 \mu \mathrm{m}$ wide ridges.

mounted junction-side-down on diamond heatsinks and have a $5 \mu \mathrm{m}$ wide ridge. In our numerical model, $R$ is proportional to the inverse of the device length. This is represented by the dashed curve in Fig. 6 .

The agreement between numerical and experimental results is excellent for devices shorter than $300 \mu \mathrm{m}$. This is not always the case for longer lasers where we observe large variations from one device to another. Before analyzing this discrepancy, it is important to point out that physical parameters such as specific heat and thermal conductivity are sensitive to stoichiometry and are not known very accurately. Thus differences between the "ideal" numerical model and the real experimental laser are unavoidable.

The differences between numerical and experimental results can have various sources. Owing to the ridge structure, the bonding of a laser junction-side-down on a heatsink is a difficult process. Nonuniformity in the soldering layer can give rise to voids which lead to hot spots and therefore increase the thermal resistance [3], and the longer the laser, the more important this effect is. We believe that this is the most important source of disagreement between experimental and numerical results. It also explains why we observe substantial variations of $\boldsymbol{R}$ between similar devices. This is not the case in the junctionside-up configuration where our results are very homogeneous [Fig. 3(a)].

Another source of discrepancy is the spontaneous emission. This radiation is isotropic and most of it is absorbed at the interface between cladding and substrate. Therefore this region acts as a second heat source. For junction-sidedown mounting, this heat source is more distant from the heatsink than the active region is and it increases the thermal resistance, although in this case the spreading of the heat flow within the GaAs substrate could partially limit this effect [4]. The light absorbed at the GaAs contact layer also acts as an additional heat source. However, its influence is effectively dumped into the close heatsinking material. In addition, part of the isotropic spontaneous emission could be absorbed at the nitride layer and act as a heat source as well. But due to the thin $\mathrm{Si}_{3} \mathrm{~N}_{4}$ layer, most of this light is reflected at the subsequent metallization layer. So the main additional contribution to $\boldsymbol{R}$ due to absorption of the spontaneous emission remains the GaAs substrate interface.

Other types of heat sources could have been considered, for example joule heating at the $p$ cladding. The electrical resistance of our lasers is typically $3 \Omega$ for a 5 $\times 500 \mu \mathrm{m}^{2}$ ridge laser and its effect should be small in the junction-side-down configuration because of the proximity of the $\mathrm{p}$ cladding with the heatsink.

\section{F. Transient Temperature}

One of the great advantages of our numerical approach is that it predicts the dynamic behavior of the system. Therefore, it is possible to calculate not only the steady state of the device but also its transient temperature. Hence one can simulate what happens thermally when a laser is switched on.

Time-resolved measurement of the temperature rise in the laser is a difficult experiment which requires a complex setup. It can be achieved, for example, by using a monochromator and a streak camera to measure the wavelength shift as a function of time. It is also possible to get a very good insight into this phenomena by simply pulsing the laser and measuring the wavelength shift as a function of the pulse length. This shift corresponds to the average of the temperature rise over the pulse length. As the measurement time is longer than one single pulse, the rise of temperature is effectively averaged over many successive pulses. To limit the parasitic heating of the device due to the successive pulses, we have used a very low duty cycle of $1: 100$.

Pulsed operation of the laser can easily be simulated with out method by switching the heat sources on and off as the calculation proceeds. We have computed the average of the temperature rise in the active region over 50 pulses with a duty cycle of $1: 100$. The results of simulations for pulse lengths between $0.1 \mu \mathrm{s}$ and $20 \mu \mathrm{s}$ are compared in Fig. 7 with the corresponding experimental results. When the laser starts to operate, the temperature rises very rapidly (on the nanosecond time scale) until it reaches the steady state, i.e., a constant temperature. For the junction-side-down configuration, it takes $0.6 \mathrm{~ms}$ to reach steady state and $3 \mathrm{~ms}$ for the junction-side-up configuration. The agreement between our experimental and numerical results is excellent as can be seen in Fig. 7 . This emphasizes the versatility of our numerical method.

\section{G. Heat Propagation Delay}

We have observed that the heatsinking material had no influence on the active region temperature during the first $600 \mathrm{~ns}$ of operation. This is easily understood from its analogy with the problem of diffusion. The diffusion of particles within a medium and the conduction of heat are identical phenomena, governed by the same equation. When particles are produced in a certain point, their diffusion is first determined by the properties of the medium 


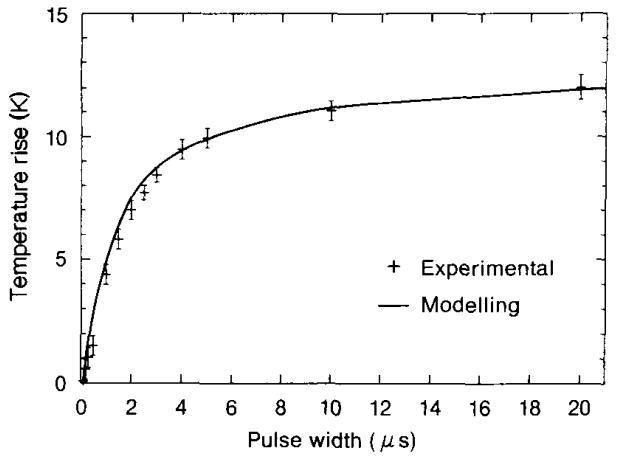

Fig. 7. Average temperature rise in the active region when the laser is pulsed, as a function of the pulse length, at a $1: 100$ duty cycle.

close to the source. The surrounding medium becomes important later, when the particles diffuse further. This is analogous for heat conduction, and it takes some time until heat reaches the heatsink. A few of these "round trip times" are necessary until the temperature in the active region is influenced by the heatsinking material.

The fact that the diffusion of heat is not instantaneous is clearly visible in Fig. 8. It presents the evolution of the temperature in the center of the structure at the laser/heatsink interface (point $\alpha$ in Fig. 1) and $12.5 \mu \mathrm{m}$ below point $\alpha$, i.e., in the heatsink (point $\beta$ in Fig. 1). The temperature at the interface starts to rise only $140 \mathrm{~ns}$ after the laser is switched on. This delay corresponds to the time needed by the heat to diffuse from the source to the top of the heatsink. It is independent of the heatsinking material.

To get a good understanding of the heat flow in a laser, it is useful to calculate the time needed by the heat to reach a given point. This heat propagation delay can be derived from numerical simulations like ours. But it can also be obtained from a simple formula. Using an instantaneous source (i.e., a finite quantity of heat instantaneously liberated at a given point) one can show that for a homogeneous medium, the average distance $\langle d\rangle$ of the heat from the source is given by [23]

$$
\langle d\rangle^{2}=\frac{6 \cdot k \cdot t}{\rho \cdot C_{p}}
$$

where heat is liberated at time $t=0$.

In our case, the medium between heat source and interface laser/heatsink (point $\alpha$ ) is not homogeneous: the heat has to flow successively through $1 \mu \mathrm{m}$ AlGaInP, 0.2 $\mu \mathrm{m} \mathrm{GaAs}$ and $2.5 \mu \mathrm{m}$ of solder (Fig. 1). Nevertheless, (5) gives a very good approximation of the time necessary to diffuse through these layers if one adds the successive values obtained for each material. This yields a delay of $132 \mathrm{~ns}$ whereas the value obtained from the simulations is $140 \mathrm{~ns}$ (Fig. 8).

An additional delay is needed for the heat to reach a point $12.5 \mu \mathrm{m}$ below the interface (point $\beta$ ). This delay now depends on the heatsinking material (Fig. 8).

From our data, one measures a delay of $30 \mathrm{~ns}$ for dia-

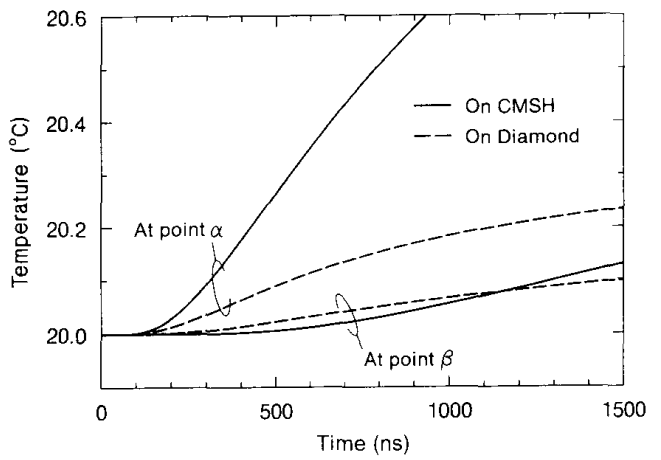

Fig. 8. Evolution of the temperature as a function of time at the laser/ heatsink interface (point $\alpha$ in Fig. 1) and $12.5 \mu \mathrm{m}$ below point $\alpha$, i.e., in the heatsink (point $\beta$ in Fig. 1). Two different heatsinking materials are investigated.

mond and $300 \mathrm{~ns}$ for CMSH. This difference stems from the difference of thermal diffusivity for these two materials. Indeed, (5) gives the following values for the time necessary to diffuse through $12.5 \mu \mathrm{m}$ of the corresponding material: $23 \mathrm{~ns}$ for diamond and $288 \mathrm{~ns}$ for CMHS. Again the agreement between this simple theoretical model and our simulations is excellent.

\section{Conclusion}

Our results show that various parameters influence the thermal resistance $\boldsymbol{R}$ of visible light AlGaInP-GaInP lasers. $\boldsymbol{R}$ is inversely proportional to the heatsink conductivity, both for junction-side-up and junction-side-down mounting. Junction-side-down mounting of red emitting laser diodes results in a marked improvement of $\boldsymbol{R}$.

The behavior of $\boldsymbol{R}$ versus the active region width depends on the mounting configuration. For junctionside-up, it is proportional to the logarithm of the width while for junction-side-down it is proportional to the inverse of the width. But in both configurations, narrow ridges are very adverse from a thermal point of view.

The soldering material has a weak influence on $\boldsymbol{R}$ and its thickness is a more critical parameter than its conductivity. On the other hand, the effect of a heat spreading gold layer on top of the laser is very favorable.

Studying the dynamic behavior of the system, we have shown that local heating phenomena occur on a nanosecond time scale while the steady state of the device is reached in the millisecond range. A simple formula has been proposed to calculate the speed of the heat flow in a laser. Finally, our experimental results have shown a very good agreement with our numerical data.

\section{ACKNOWLEDGMENT}

We are very thankful to R. F. Broom who provided the physical parameters used in the simulations and to H.P. Gschwind and H. Richard for technical support. It is also a pleasure to acknowledge valuable discussions with $\mathrm{L}$. R. Brovelli, H. Jaeckel, P. Roentgen, and P. Unger. 


\section{REFERENCES}

[1] W. B. Joyce and R. W. Dixon, "Thermal resistance of heterostructure lasers," J. Appl. Phys., vol. 46, pp. 855-862, 1975

[2] T. Kobayashi and Y. Funkawa, "Temperature distribution in the GaAS-AlGas, double-heterostructure laser below and above the threshold current,' Japan. J. Appl. Phys., vol. 14, pp. 1981-1986, 1975.

[3] T. Kobayashi and G. Iwane, "Three dimensional thermal problems of double-heterostructure semiconductor lasers," Japan. J. Appl. Phys., vol. 16, pp. 1403-1408, 1977.

[4] D. H. Newman, D. J. Bond, and J. Stefani, "Thermal-resistance models for proton-isolated double-heterostructure lasers," Solid State Electron Devices, vol. 2, pp. 41-46, 1978.

[5] E. Duda, J.-C. Carballes, and J. Apruzzese, "Thermal resistance and temperature distribution in double-heterostructure lasers: Calculations and experimental results," IEEE J. Quantum Electron., vol. QE-15, pp. 812-817, 1979.

[6] M. Ito and T. Kimura, "Stationary and transient thermal propertie of semiconductor laser diodes," IEEE J. Quantum Electron., vol. QE-17, pp. 787-795, 1981.

[7] H. C. Hsieh, "Maximum heat-sink temperature for CW operation of a double-heterostructure semiconductor injection laser," IEEE $J$. Quantum Electron., vol. 25, pp. 2079-2083, 1989.

[8] W. Nakwaski, "Three-dimensional analysis of a heat-spreading phenomenon in phase-locked arrays of oxide-isolated diode lasers," $J$. Appl. Phys., vol. 67, pp. 2711-2715, 1990.

[9] Y. Ueno, K. Endo, H. Fujii, K. Kobayashi, K. Hara, and T. Yuasa, "Continuous-wave high-power $(75 \mathrm{~mW})$ operation of a transversemode stabilised window-structure $680 \mathrm{~nm}$ AlGaInP visible diode," Electron. Lett., vol. 26, pp. 1726-1728, 1990.

[10] G. L. Bona, P. Roentgen, P. Unger, and H. Jaeckel, “High quality index guided ridge $\mathrm{AlGaInP/GaInP}$ visible lasers," in Proc, 12th IEEE Int. Semicond. Laser Conf., Davos, Switzerland, Sept. 9-14, 1990, paper L2, pp. 178-179.

[11] M. Ishikawa, H. Okuda, K. Itaya, H. Shiozawa, and Y. Uematsu, "Long-term reliability tests for InGaAlP visible laser diodes," $\mathrm{Ja}$ pan. J. Appl. Phys., vol. 28, pp. 1615-1621, 1989.

[12] G. Hatakoshi, M. Suzuki, N. Motegi, M. Ishikawa, and Y. Uematsu "Thermal analysis for InGaAlP laser diodes," Trans. IEICE, vol. E 71, pp. 315-317, 1988 .

[13] S. Adachi, "GaAs, AlAs and $\mathrm{Al}_{x} \mathrm{Ga}_{1-x} \mathrm{As}$ : Material parameters for use in research and device applications," J. Appl. Phys., vol. 58, pp. R1-R29, 1985.

[14] "Metals and materials for research and industry, catalogue 1989," Goodfellow Metals Ltd., England.

[15] "CMSH," Sumitomo Electric Industries Ltd., Japan, 1986.

[16] S. M. Sze, VLSI Technology, 2nd ed. Singapore: McGraw-Hill Int., 1988.

[17] J. Ligou, "Troisième cycle de la physique en Suisse Romande: Expérimentation numérique," Swiss Federal Institute of Technology, Lausanne, 1988

[18] H. Okuda, M. Ishikawa, H. Shiozawa, Y. Watanabe, K. Itaya, K. Nitta, G.-I. Hatakoshi, Y. Kobubun, and Y. Uematsu, "Highly reliable InGaP/InGaAlP visible light emitting inner stripe lasers with $667 \mathrm{~nm}$ lasing wavelength," IEEE J. Quantum Electron., vol. 25, pp. $1477-1482,1989$.

[19] Y. Takahashi, M. Mannoh, J.-I. Hoshina, S. Kamiyama, Y. Sasai, K. Ohnaka, and M. Ogura, "A new index-guided AlGaInP visible semiconductor laser with very small astigmatism," IEEE Trans. Electron Devices, vol. 36, p. 2607, 1989.

[20] P. Wolf, unpublished.

[21] T. L. Paoli, "A new technique for measuring the thermal impedance of junction lasers," IEEE J. Quantum Electron., vol. QE-11, pp. 498-503, 1975.

[22] O. J. F. Martin, unpublished.

[23] H. S. Carslaw and J. C. Jaeger, Conduction of heat in solids, 2nd ed. London: Oxford, 1959, ch. 10.

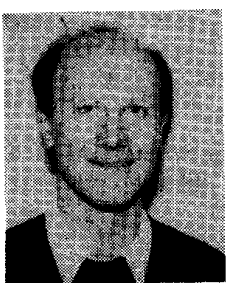

Olivier J. F. Martin received the Diplôme d'Ingénieur Physicien from the Swiss Federal Institute of Technology, Lausanne, Switzerland, in 1989. His diploma thesis dealt with the electronics prop erties of high $T_{c}$ superconductors.

In June 1989, he joined the Laser Science and Technology Department of the IBM Zurich Research Laboratory, where he is presently working toward the Ph.D. degree in physics. His research interests include computational optoelectronics with an emphasis on thermal and optical properties of semiconductor lasers.

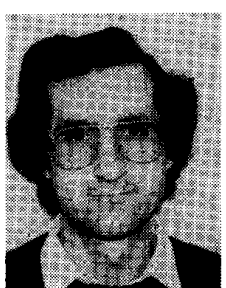

Gian-Luca Bona received his undergraduate and Ph.D. degrees in physics in 1982 and 1988, respectively, from the Swiss Federal Institute of Technology (ETH), Zurich, Switzerland. His dissertation was on an investigation of the surface magnetic properties of magnetooptic materials by short pulsed laser excited photoemission.

In 1987, he joined the IBM Zurich Research Laboratory, where he was involved in ps optical sampling of ultrafast devices, in particular interdigitated photodetectors. Since 1988, he has been working on $\mathrm{AlGaAs}$ high-power quantum-well lasers for optical storage applications. Currently, his research interests are focused on device design and characterization of $\mathrm{AlGaInP}-\mathrm{GaInP}$ visible laser diodes.

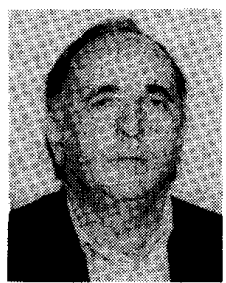

Peter Wolf received the B.S. degree from the University of Karlsruhe, Germany, in 1953, the M.S. degree from the University of Darmstadt, Germany, in 1958, and the Ph.D. degree from the University of Mainz, Germany, in 1963, all in physics.

In 1959, he joined the IBM Zurich Research Laboratory, where he has worked on the spin dynamics of thin magnetic films, the dynamics of spin structures in rare-earth metals, microwave Schottky-barrier field-effect transistors (MESFET's) and applications of Josephson junctions in memories and high speed samplers. Currently, he is manager of a devices group and since 1984 has been engaged in optoelectronic applications of III-V devices.

Dr. Wolf is a member of the German Physical Society. 